\title{
High-resolution THz spectrometer with kHz scan rates
}

\author{
A. Bartels ${ }^{1,2}$, A. Thoma ${ }^{1}$, C. Janke ${ }^{1,2}$, T. Dekorsy ${ }^{1}$, A. Dreyhaupt ${ }^{3}$, S. \\ Winnerl ${ }^{3}$, and M. Helm ${ }^{3}$ \\ ${ }^{1}$ University of Konstanz and Center for Applied Photonics, Universitätsstrasse 10, 78457 \\ Konstanz, Germany \\ ${ }^{2}$ Gigaoptics GmbH, Blarerstrasse 56, 78462 Konstanz, Germany \\ ${ }^{3}$ Institute of Ion Beam Physics and Materials Research, Forschungszentrum Rossendorf, P.O. \\ Box 510119, 01314 Dresden, Germany \\ bartels@gigaoptics.com \\ http://www.gigaoptics.com
}

http://www.uni-konstanz.de/FuF/Physik/dekorsy

\begin{abstract}
We demonstrate a rapid scanning high-resolution $\mathrm{THz}$ spectrometer capable of acquiring $\mathrm{THz}$ field transients with $1 \mathrm{~ns}$ duration without mechanical delay line. The $\mathrm{THz}$ spectrometer is based on two 1-GHz Ti:sapphire femtosecond lasers which are linked with a fixed repetition rate difference in order to perform high-speed asynchronous optical sampling. One laser drives a high-efficiency large-area GaAs based $\mathrm{THz}$ emitter, the other laser is used for electro-optic detection of the emitted THz-field. At a scan rate of $9 \mathrm{kHz}$ a time resolution of $230 \mathrm{fs}$ is accomplished. High-resolution spectra from $50 \mathrm{GHz}$ up to 3 $\mathrm{THz}$ are obtained and water absorption lines with a width of $11 \mathrm{GHz}$ are observed. The use of femtosecond lasers with $1 \mathrm{GHz}$ repetition rate is essential to obtain rapid scanning and high time-resolution at the same time.
\end{abstract}

OCIS codes: (320.7150) ultrafast spectroscopy; (320.7090) ultrafast lasers.

\section{References and links}

1. D. Mittleman (ed.), Sensing with THz radiation (Springer, Heidelberg, 2002) and references therein.

2. C. Janke, M. Först, M. Nagel, H. Kurz, and A. Bartels, "Asynchronous optical sampling for high-speed characterization of integrated resonant THz sensors," Opt. Lett. 30, 1405-1407 (2005).

3. T. Yasui, E. Saneyoshi, and T. Araki, "Asynchronous optical sampling THz time-domain spectroscopy for ultrahigh spectral resolution and rapid data acquisition," Appl. Phys. Lett. 87, 061101 (2005).

4. P.A. Elzinga, F.E. Lytle, Y. Jian, G.B. King, and N.M. Laurendeau, "A pump/probe method for fast analysis of visible spectral signatures utilizing asynchronous optical sampling," Appl. Opt. 264303 (1987).

5. F. Keilmann, C. Gohle, and R. Holzwarth, "Time-domain mid-infrared frequency-comb spectrometer," Opt. Lett. 29 1542-1544 (2004).

6. F.C. DeLucia, "Noise, detectors, and submillimeter-terahertz system performance in nonambient environments," J. Opt. Soc. Am. B 21 1273-1279 (2004).

7. D.W. van der Weide, J. Murakowski, and F. Keilmann, "Gas-absorption spectroscopy with electronic terahertz techniques," IEEE Trans. Microwave Theory Tech. 48 740-743 (2000).

8. A. Bartels, F. Hudert, C. Janke, T. Dekorsy, and K. Köhler, "Femtosecond time-resolved optical pump-probe spectroscopy at kHz-scan-rates over ns-time-delays without mechanical delay line," Appl. Phys. Lett. submitted (2005).

Konstanzer Online-Publikations-System (KOPS)

URL: http://www.ub.uni-konstanz.de/kops/volltexte/2008/4546/

URN: http://nbn-resolving.de/urn:nbn:de:bsz:352-opus-45464 
9. A. Dreyhaupt, S. Winnerl, T. Dekorsy, and M. Helm, "High-intensity THz radiation from a microstructured large-area photoconductor," Appl. Phys. Lett. 86121114 (2004).

10. Q. Wu and X.-C. Zhang, "Ultrafast electro-optic field sensors," Appl. Phys. Lett. 68 1604-1606 (1996).

11. R. Huber, A. Brodschelm, F. Tauser, and A. Leitenstorfer, "Generation and field resolved detection of femtosecond electromagnetic pulses tunable up to 41 THz," Appl. Phys. Lett. 76 3191-3193 (2000).

12. H.J. Bakker, G.C. Cho, H. Kurz, Q. Wu, and X.-C. Zhang, "Distortion of THz pulses in electro-optic sampling," J. Opt. Soc. Am. B 15 1795-1801 (1998).

13. P. Planken, N. Nienhuys, H.J. Bakker, and W.T. Wenckebach, "Measurement and calculation of the orientation dependence of terahertz pulse detection in ZnTe," J. Opt. Soc. Am. B 18 313-317 (2001)

14. D.E. Burch, "Absorption of infrared radiant energy by $\mathrm{CO}_{2}$ and $\mathrm{H}_{2} \mathrm{O}$. III. Absorption of $\mathrm{H}_{2} \mathrm{O}$ between 0.5 and $36 \mathrm{~cm}^{-1}(278 \mu \mathrm{m}-2 \mathrm{~cm})$, , J. Opt. Soc. Am. 58 1383-1394 (1968).

15. J.M. Flaud, C. Camy-Peyret, and R.A. Toth, Water vapor line parameters from microwave to medium infrared (Pergamon, Oxford, 1981).

16. M. van Exter, Ch. Fattinger, and D. Grischkowsky, "Terahertz time-domain spectroscopy of water vapor," Opt. Lett. 14 1128-1130 (1989).

\section{Introduction}

The generation and detection of $\mathrm{THz}$ radiation with femtosecond lasers is a rapidly evolving field due its relevance for the optical characterization of a wide variety of material systems [1]. Conventional THz time-domain spectroscopy (THz-TDS) systems are based on pulsed $\mathrm{THz}$ radiation that is repetitively generated by a modelocked train of femtosecond laser pulses in a photoconductive switch, via optical rectification or difference frequency mixing. A second time-delayed pulse train originating from the same laser is used to detect the THz electric field in amplitude and phase via a photoconductive antenna or the electro-optic effect. To achieve a high frequency resolution on the order of $1 \mathrm{GHz}$, a mechanical delay line providing $1 \mathrm{~ns}$ optical time delay (corresponding to a mechanical movement of $2 \times 15 \mathrm{~cm}$ ) must be used. Since mechanical delay generators are limited in scanning speed, they provide a basic limit for scan rates in THz-TDS and hence limit the signal-to-noise ratio achievable in a given measurement time. Recently, concepts for THz-TDS without mechanical delay generators and potentially higher scanning rates have been demonstrated $[2,3]$. They employ two separate ultrafast laser sources that are operated with a small difference frequency $\Delta f_{R}$. The separate lasers provide two pulse trains whose time-delay is linearly ramped between zero and $f_{R}^{-1}$ at a rate given by $\Delta f_{R}$. This concept is generally referred to as asynchronous optical sampling (ASOPS) [4]. In related work, two modelocked femtosecond lasers have been employed to eliminate the mechanical delay line from a mid-infrared Fourier-transform spectrometer [5]. The device does not detect $\mathrm{THz}$ electric field transients directly but is based on an interferogram between the THz radiation emitted from two GaSe crystals via optical rectification. The nonlinear crystals were driven by separate Kerr-lens modelocked Ti:sapphire femtosecond lasers with repetition rates of $\approx 87$ $\mathrm{MHz}$ repetition rate and a small relative offset of $\Delta f_{R}=2 \mathrm{~Hz}$. Interferograms were acquired at a rate of $2 \mathrm{~Hz}$ without moving delay scanner as would be required for a conventional FTIR spectrometer or a conventional THz-TDS system. The demonstrated bandwidth from $22.5 \mathrm{THz}$ to $39 \mathrm{THz}$ was only limited by the pulse duration of the femtosecond lasers ( $20 \mathrm{fs}$ and $12 \mathrm{fs}$ ). However, the reproducibility and frequency resolution was limited by slow drifts of the uncontrolled laser repetition rates. Yasui and coworkers demonstrated a THz-TDS system based on two Kerr-lens mode-locked Ti:sapphire lasers with $f_{R}=82.6 \mathrm{MHz}$ and a scan rate of $\Delta f_{R}=108$ $\mathrm{Hz}$ [3]. In their set-up $10 \mathrm{fs}$ and $60 \mathrm{fs}$ pulses were used for generation and detection of THz radiation in photoconductive antennas, respectively. The detected $\mathrm{THz}$ spectrum covered the range from $20 \mathrm{GHz}$ to $120 \mathrm{GHz}$, limited by the jitter of the laser stabilization. A frequency resolution of $82.6 \mathrm{MHz}$ was proposed in view of the laser repetition rate, however, no such spectral line width was observable in the measured $\mathrm{THz}$ spectrum. In the lower $\mathrm{THz}$ frequency range, the latter work competes with existing all-electronic $\mathrm{cw}$ submillimeter techniques which provide 
high resolution in the $\mathrm{MHz}$ range and scanning rates in the range of $10 \mathrm{GHz} / \mathrm{s}[6,7]$. To fully exploit the advantages of the discussed approaches for THz-TDS in terms of acquisition speed, frequency bandwidth and resolution, it is obvious that higher scan rates and better repetition rate stabilization are required. The field of THz-TDS without mechanical delays and high scan rates has recently been much advanced by high-speed ASOPS systems based on two Kerr-lens mode-locked Ti-sapphire lasers with $f_{R}=1 \mathrm{GHz}$ and a scan rate $\Delta f_{R}$ in the $\mathrm{kHz}$ frequency range. Such systems were demonstrated for the detection of electrical transients on integrated coplanar resonators [2] and all-optical pump-probe spectroscopy [8]. The former system permitted spectroscopy up to $500 \mathrm{GHz}$ [2], while the latter provided an enhanced bandwidth up to $4 \mathrm{THz}$ due to an improved laser stabilization scheme [8]. Laser repetition rates in the range of $1 \mathrm{GHz}$ are generally favorable for ASOPS in order to achieve a sub-picosecond time-resolution and at the same time a high scan rate in the multi-kHz regime, since they reduce the required optical detection and data acquisition bandwidth $F$ to practically accessible values in the range of 100 $\mathrm{MHz}$.

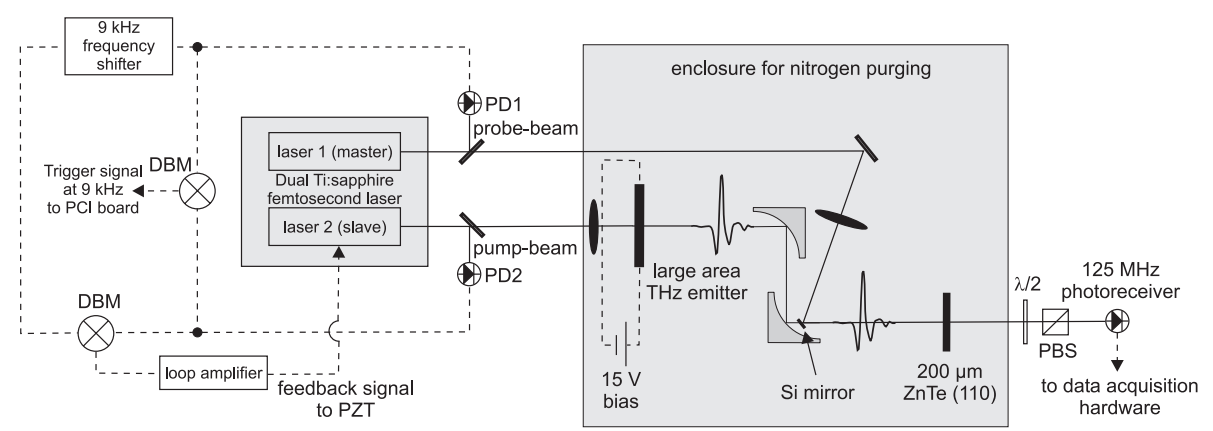

Fig. 1. Experimental setup. Solid lines are optical paths, dashed lines represent electronic signals. See text for details.

Here, we present a rapid-scanning high-resolution $\mathrm{THz}$ spectromenter based on a dual laser system that comprises two Ti:sapphire femtosecond oscillators with $1 \mathrm{GHz}$ repetition rate and active offset frequency stabilization (Gigajet TWIN and stabilization unit TL-1000, Gigaoptics $\mathrm{GmbH}$, Germany). THz absorption spectra of water vapor at frequencies up to $3 \mathrm{THz}$ are obtained with a dynamic range of $30 \mathrm{~dB}$ within a total measurement time of only $250 \mathrm{~s}$.

\section{Experiment}

The two femtosecond Ti:sapphire oscillators with repetition rates $f_{R, 1}$ and $f_{R, 2}$ of approximately $1 \mathrm{GHz}$ are pumped by a diode-pumped solid state laser at $532 \mathrm{~nm}$ wavelength whose $6.5 \mathrm{~W}$ output power is split into equal portions. The oscillators deliver approximately $500 \mathrm{~mW}$ of output power with a pulse duration of $30 \mathrm{fs}$. The repetition rate difference $\Delta f_{R}=f_{R, 1}-f_{R, 2}$ is fixed using active feedback. The time-delay between the two pulse trains is then $\tau(t)=$ $t \times \Delta f_{R} / f_{R, 2}$ modulo $f_{R, 2}^{-1}$ if laser 2 is used as the pump-laser and $t$ is the real-time. The quality of the feedback electronics determines the accuracy of the $\tau(t)$ relationship given above through a jitter of the time base which directly determines the experimental time resolution. Instead of employing two feedback loops to stabilize the lasers to their individual reference synthesizers with an offset of $\Delta f_{R}$ [2], we use laser 1 as master-laser and link laser 2 as slave-laser to it at a difference of $\Delta f_{R}=9 \mathrm{kHz}$. This has the advantage that only the residual errors of one feedback 
loop enter the jitter of the time-base.
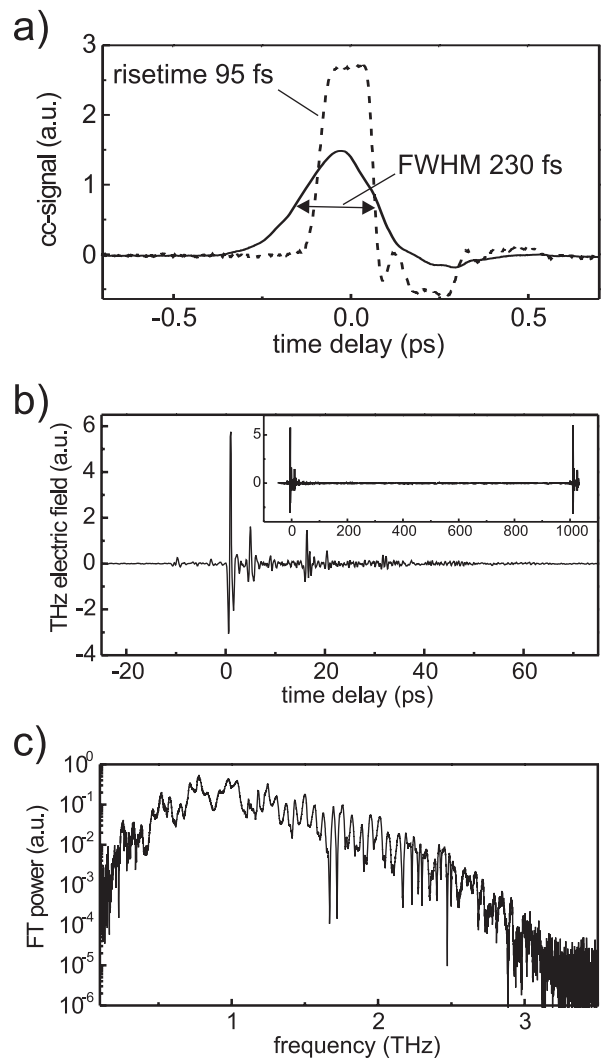

Fig. 2. a) Cross-correlation signal between lasers 1 and 2: single scan (dashed line) and averaged over 512 scans (solid line). b) Zoomed view of a THz transient acquired under nitrogen purging of the setup with $N=10000$ and a total acuisition time of 2500 s. The inset shows the entire data set where the signal is repeated after an inverse repetition rate of laser 2. c) Fourier-transform spectrum of the time-domain signal displayed in b).

The experimental scheme is sketched in Fig. 1 . We measure $f_{R, 1}$ by detecting laser 1 with a fast photodiode (PD1) and upshift the frequency of the electronic signal by $9 \mathrm{kHz}$ using a frequency-shifter. The frequency-shifter consists of a single-sideband generator with a carrier suppression stage that allows a suppression of the initial carrier signal by better than 50 $\mathrm{dB}$. Subsequently, $f_{R, 2}$ is detected with a second photodiode (PD2) and phase-locked to the frequency-shifted repetition rate signal of laser 1. To accomplish this, we use a double-balanced mixer (DBM) as phase-detector and feed its output back to a piezoelectric transducer that supports a cavity mirror in laser 2 via an adjustable loop-amplifier. The jitter of the time-base $\tau(t)$ is characterized by detecting a cross-correlation signal between the two lasers using noncollinear sum-frequency generation in a $\beta$-barium-borate-crystal. The cross-correlation signal is detected with a $125-\mathrm{MHz}$ bandwidth photoreceiver and recorded using a sampling oscilloscope (Tektronix TDS3032B). To generate a trigger signal for the oscilloscope at $\Delta f_{R}$, we use a second DBM with the signals from PD1 and PD2 as inputs. The real-time scale is converted to a time-delay scale by applying a factor $\Delta f_{R} / f_{R, 2}$. Figure 2(a) shows a single scan measurement of the cross-correlation signal and an average over 512 measurements acquired over $\approx 1$ $\mathrm{s}$, limited by the averaging function of the oscilloscope. The single scan trace with a rise time 
of $95 \mathrm{fs}$ shows the limitation to the time-resolution $\Delta \tau$ given by the $100 \mathrm{MHz}$ bandwidth of the sampling oscilloscope (determined by our specific chosen acquisition settings) according to $\Delta \tau=\Delta f_{R} / f_{R, 2} F$, where $F$ is the effective bandwidth of the photodetection and data acquisition electronics. The detailed structure is attributed to the response of the photoreceiver. The averaged cross-correlation signal has a width of $230 \mathrm{fs}$, reflecting residual jitter of the timebase. Averaging over longer times does not further broaden the signal, thus 230 fs represents the overall time-resolution of our setup. This limit is mostly due to phase-noise intrinsic to the DBMs used for phase-detection and trigger-generation as well as a residual contribution at the carrier frequency at the output of the frequency shifter. We envision that an improvement of the timing stability to the sub-100 fs regime is possible by performing the phase-lock at a higher harmonic of the repetition rate or by using digital phase-detectors.

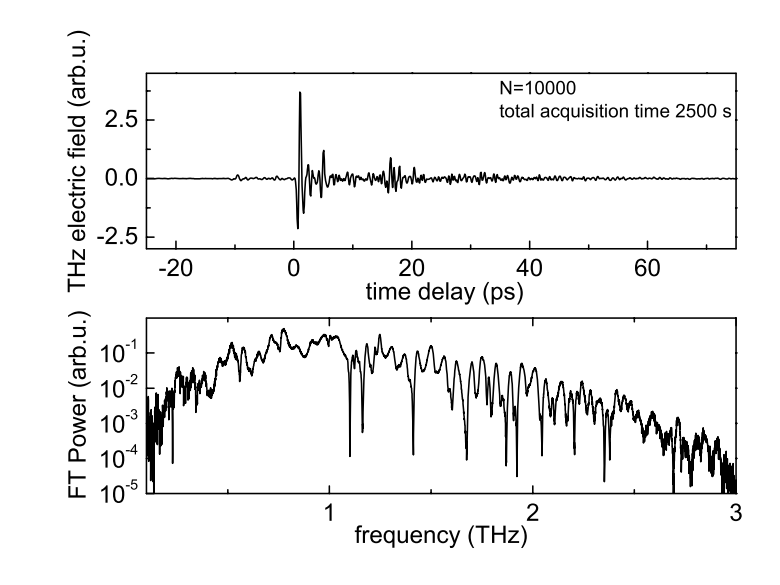

Fig. 3. THz transients with water vapor present in the setup at different $N$ and Fourier transform spectra (series of 5 animated frames).

Laser 2 drives the THz emitter device which is composed of a large-area interdigitated finger structure on a semi-insulating GaAs substrate [9]. Optical excitation is performed under normal incidence with an average power of $400 \mathrm{~mW}$. The interdigitated finger structure has an electrode spacing of $5 \mu \mathrm{m}$ and is biased with $15 \mathrm{~V}$ of DC voltage which results in an emission bandwidth of approximately $2.5 \mathrm{THz}$ at $10 \mathrm{~dB}$ below the maximum. The photocurrent through the emitter is $35 \mathrm{~mA}$. The advantage of this emitter in comparison to photoconductive antennas with small electrode gap spacing is the possibility to dissipate a greater excitation power [9]. The emitter efficiency is approximately $10^{-4}$ corresponding to $40 \mu \mathrm{W}$ of average $\mathrm{THz}$ power. The $\mathrm{THz}$ pulse is emitted through the GaAs substrate and collected with a $90^{\circ}$ off-axis parabolic mirror with a $10 \mathrm{~cm}$ focal length. It is re-focused with another $10 \mathrm{~cm}$ focal length off-axis paraboloid for detection. The detection of the $\mathrm{THz}$ pulse is based on the electro-optic effect in a (110) ZnTe crystal of $200 \mu \mathrm{m}$ thickness [10,11]. The probe pulse is focused to the same spot as the THz pulse on the detector crystal by using a small piece of Si substrate as a mirror of a few square millimeters area close to the second off-axis paraboloid. This arrangement does not block or modify most of the THz radiation. The probe laser wavelength can be continuously tuned to provide optimum phase matching between the optical and the THz pulse in the crystal [12]. Experiments are performed at a probe wavelength of $830 \mathrm{~nm}$. The probe beam polarization behind the ZnTe crystal is analyzed with a half-wave plate $(\lambda / 2)$ and a polarizing beam splitter (PBS). Instead of using the common scheme where the difference between perpendicular polarizations is detected, we place a single photoreceiver in one of the beams behind the 
beam splitter to obtain the electro-optic signal. Compared to the conventional approach, we loose a factor of two in signal amplitude but circumvent bandwidth limitations introduced by available difference amplifiers. The photoreceiver is AC coupled and has a bandwidth of 125 MHz. Its output is directly supplied to a $200 \mathrm{Ms} / \mathrm{s} 14 \mathrm{Bit} \mathrm{A} / \mathrm{D}$ converter on a PCI-board which is triggered by the synchronization electronics. The data acquisition board has the capability of acquiring hardware-based averages over 1024 single scan traces. These averaged traces are displayed at a rate of $4 \mathrm{~Hz}$ on the computer. An arbitrary number of additional software based averages can be computed. The spatial overlap of THz- and probe-pulse, the optimum angle of laser polarization and the ZnTe crystal orientation for $\mathrm{THz}$ detection [13] can be optimzed 'online' by observing the electro-optic THz signal transient which is displayed almost at a video rate on our data acquisition computer. In comparison to conventional THz-TDS systems with mechanical delay lines no temporal overlap needs to be adjusted or tended during optimization. The bandwidth of the entire system is currently limited by the phasematching bandwidth of the ZnTe crystal at our detection wavelength of $830 \mathrm{~nm}$.

\section{Results and discussion}

We have acquired THz transients with different numbers of software averages $N$ such that the total number of averaged single scan traces is $N \times 1024$. The acquisition time for each data set amounts to $N \times 0.25 \mathrm{~s}$. A measurement with $N=10000$, acquired under purging of the setup with nitrogen, is displayed in Fig. 2(b). A weak signal background, acquired with unbiased THz emitter, has been subtracted from all data. The origin of the signal background is scattered light reaching the photoreceiver from laser 2 which has a slight power modulation at $\Delta f_{R}$ due to imperfections of the frequency shifter. The time-domain signal shows a strong $\mathrm{THz}$ pulse with weaker pre- and postpulses that are due to multiple reflections of the optical beams off uncoated glass substrates that are used as beamsplitters in the setup as well as a multiple reflection of the THz pulse off the emitter substrate. These pre- and post-pulses cause the modulations of the Fourier-transform spectrum shown in Fig. 2(c). A series of measurements with water vapor present in the setup at different values of $N$ is displayed in Fig. 3, where the time window has been limited to $100 \mathrm{ps}$ for better visibility. Fourier-transform spectra have been computed from the full data sets (covering $1 \mathrm{~ns}$ ) and are displayed with the THz transients. The bandwidth of the spectra above the noise level amounts to $3 \mathrm{THz}$ at $N \geq 1000$. The signal-to-noise ratio obtained for the Fourier-transform spectra increases linearly with total acquisition time and amounts to $\approx 50 \mathrm{~dB}$ at $N=10000$. From a more detailed analysis we conclude that signals are at the detection shot-noise limit.

From the datasets with and without water vapor present in the setup, we have computed water absorption spectra as shown in Fig. 4. Lines at frequencies up to $3 \mathrm{THz}$ are clearly identified at $N=100$ (i.e. only $25 \mathrm{~s}$ of measurement time per data set) and are readily observed with a dynamic range of $30 \mathrm{~dB}$ with $N=1000$. All lines can be attributed to previously reported water resonances in the observed frequency range $[14,15,16]$. The full width at half maximum of the absorption resonances is approximately $11 \mathrm{GHz}$, a detailed view of the line at $558 \mathrm{GHz}$ at $N=10000$ is shown in Fig. 5. It has been shown earlier in all-optical pump-probe experiments that the resolution limit of our high-speed ASOPS approach is $1 \mathrm{GHz}$ [8]. Thus we conclude that the observed spectral lineshape is likely limited by saturation and collision broadening.

\section{Conclusion}

In conclusion, we have constructed a high-resolution $\mathrm{THz}$ spectrometer without mechanical delay line. An unprecedented scan rate of $9 \mathrm{kHz}$ is accomplished, the experimental resolution limit amounts to $1 \mathrm{GHz}$. Employing a high-power large-area $\mathrm{THz}$ emitter, we have observed $\mathrm{THz}$ transients of $1 \mathrm{~ns}$ duration with a time resolution of $230 \mathrm{fs}$. We have demonstrated the 


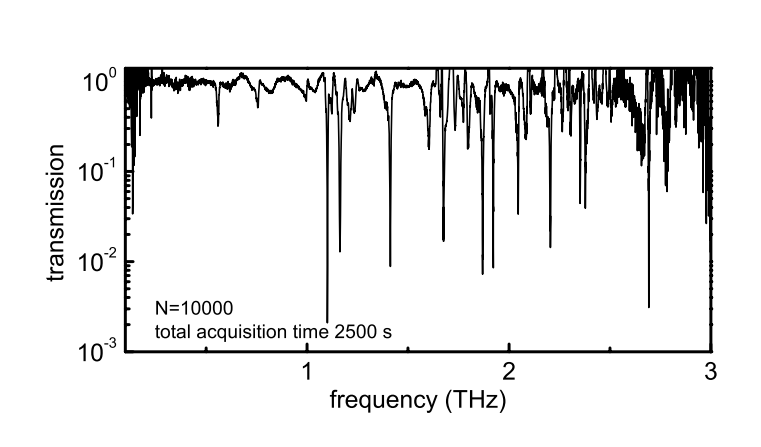

Fig. 4. Water absorption spectra computed from Fourier transforms of time-domain data under nitrogen purging and with water vapor present in the setup (series of 5 animated frames).

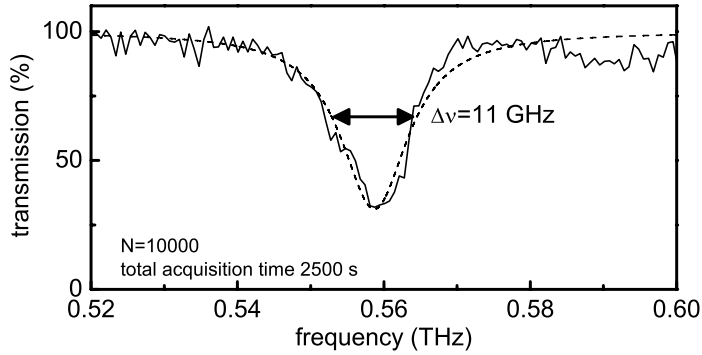

Fig. 5. Detailed view of water absorption line at $558 \mathrm{GHz}$. A Lorentzian fit with a full width at half maximum of $11 \mathrm{GHz}$ is displayed as dashed curve.

acquisition of $\mathrm{THz}$ water absorption spectra at frequencies up to $3 \mathrm{THz}$ that are acquired with a dynamic range of $30 \mathrm{~dB}$ within only $250 \mathrm{~s}$ of acquisition time per signal and reference data set. The observed width of the absorption lines is $11 \mathrm{GHz}$, likely limited by saturation and collision broadening effects. The spectrometer is based on high-speed asynchronous optical sampling employing a dual modelocked laser system with femtosecond oscillators that have approximately $1 \mathrm{GHz}$ repetition rate. The high laser repetition rates are essential in order to achieve a sub-picosecond time resolution and at the same time a high scanning rate in the multi-kHz regime. Our high-resolution $\mathrm{THz}$ spectrometer has great potential to advance the field of THz-TDS in general. It allows to significantly reduce data acquisition times for a wide variety of spectroscopic applications and thus opens the way e.g. for high-throughput monitoring of material properties and could lead to much accelerated frame acquisition in THz imaging applications. The presented system has potential for further improvements which are explored in current experiments: The time-resolution can be improved by stabilizing the repetition rates at higher harmonics. The signal-to-noise ratio can be further increased, leading to even shorter data acquisition times, by tighter focusing of the $\mathrm{THz}$ radiation and by using high-bandwidth difference amplifiers and employing conventional electro-optic detection. 


\section{Acknowledgments}

We thank A. Leitenstorfer for stimulating discussions and acknowledge contributions of S.A. Diddams, S. Eggert, and E.N. Ivanov to the frequency shifter. This work has partially been supported by the Ministry of Science, Research and the Arts of Baden-Württemberg. 